Artículo

\title{
Competitividad del tomate rojo de México en el mercado internacional: análisis 2003-2017
}

Iliana Enriqueta Montaño Méndez ${ }^{\S}$

Iván Narcizo Valenzuela Patrón

Kennia Valeria Villavicencio López

Instituto Tecnológico Superior de Mulegé. Loma de los frailes s/n, Col. Centro, Santa Rosalía, Baja California Sur, México. CP. 23920. Tel. 615 6890000. (ivan.vp@mulege.tecnm.mx; kennia.v105@gmail.com).

${ }^{\S}$ Autora para correspondencia: iliana.mm@mulege.tecnm.mx.

\section{Resumen}

En México el sector agropecuario contribuye con cerca de 3.4\% del PIB nacional, sobresaliendo la actividad hortofrutícola con $45 \%$ de las exportaciones del sector, donde el tomate rojo representa $8.41 \%$. México es el principal proveedor a nivel mundial de este producto con una participación de $19 \%$ de las exportaciones mundiales por arriba de España o Países Bajos. El objetivo es determinar la competitividad del tomate rojo de México en el mercado internacional mediante la ventaja relativa de exportación (VRE) y la participación constante de mercado (CMS). Se encontró competitividad del producto mexicano en el mercado estadounidense y una alta concentración de mercado, enviando $98 \%$ de las exportaciones de tomate rojo mexicano hacia ese destino.

Palabras claves: competitividad, exportaciones, mercado, producto agrícola.

Recibido: junio de 2021

Aceptado: septiembre de 2021 


\section{Introducción}

La agricultura en México es una actividad importante no sólo por la producción de alimentos sino también porque es generadora de empleos, de divisas y por las relaciones conexas que se tornan alrededor de la actividad (Álvarez et al., 2017). En México el sector agropecuario contribuye con cerca de $3.4 \%$ del producto interno bruto (PIB), genera $13 \%$ del empleo, registra cuatro millones de unidades de producción (INEGI, 2007), abarca una extensión de 110 millones de hectáreas, de las cuales 32 millones son para uso agrícola (CEDRSSA, 2019) y aporta $1.75 \%$ de las exportaciones agropecuarias mundiales.

La actividad hortofrutícola representa $45 \%$ de las exportaciones, ubicándola como la actividad más dinámica en términos de exportaciones a partir de la firma del Tratado de Libre Comercio de América del Norte (Avendaño y Schwentesius, 2012; Ávila y González, 2012), destacando la exportación de productos tales como aguacate $(2.4 \%)$, limones $(2.86 \%)$, cebolla $(2.23 \%)$, pepino (3.31\%), pimiento (3.9\%) y tomate (7.77\%) (FAOSTAT, 2021). El sector agrícola en México mantiene una balanza comercial superavitaria (SADER, 2021), coadyuvando a incentivar la economía nacional con la generación empleos y mayores ingresos.

México aporta $1.7 \%$ de la producción mundial de tomate, por debajo de China, China Continental, India y Estados Unidos de América, entre otros, contribuye 19\% de volumen de las exportaciones a nivel mundial, ubicándolo como el principal país exportador por arriba de España (14\%) y Países Bajos (13\%) (FAOSTAT, 2020). Durante el periodo de 2003 a 2017, en México se produjeron en promedio 2.5 millones de toneladas de tomate rojo y se generan por año 14759 millones de pesos (SIAP, 2020).

México presenta características particulares que han generado condiciones favorables para la producción y exportación de hortalizas, tales como, el clima, recursos naturales, disponibilidad de mano de obra, cercanía geográfica con el mercado estadounidense (considerado el más grande del mundo) y uso de innovaciones tecnológicas (Hernández et al., 2017; Bracamontes y Méndez, 2011; Hernández, 2019). Lo anterior, se relaciona con la ventaja comparativa de David Ricardo que determina quién producirá un bien. Krugman y Obstfeld (2006) mencionaron que, un país tiene ventaja comparativa en la producción de un bien si el coste de oportunidad en la producción de este bien en términos de otros bienes es inferior en este país de lo que es en otros países.

Aunado a ello, Pugel (2004) menciona que un país tendrá ventaja comparativa cuando exporte aquellos bienes y servicios que pueda producir a un costo de oportunidad menor e importará aquellos bienes y servicios que, de otro modo, produciría a costo de oportunidad más alto. Para la medición de la competitividad de las exportaciones en el mercado internacional se puede utilizar el índice de ventaja comparativa revelada de Vollrath (1991) mediante el índice de ventaja relativa de exportaciones y el índice de ventaja comparativa revelada. La ventaja relativa de exportación indica las ventajas de un país en las exportaciones con respecto al mundo (Ramírez et al., 2020).

Las fuentes de la ventaja comparativa pueden ser: diferencias en la dotación de factores de producción, diferencias tecnológicas o diferencias en la demanda (Macías, 2010). Se han realizado estudios para medir la competitividad de productos hortofrutícola de México en el mercado internacional como de aguacate (Torres, 2009), fresa (Ávila y González, 2012; Ramírez et al., 
2016), nuez pecanera (Ávila et al., 2020), café (Valencia, 2016), uva (Valencia y Duana, 2019), cebolla (Valencia y Espinoza, 2017), entre otros o estudios donde se estima la competitividad de productos agrícolas encontrando que las frutas y hortalizas de México son competitivas en el mercado mundial (Avendaño y Schwentesius, 2005).

El objetivo de documento es determinar la competitividad del tomate rojo de México en el mercado internacional mediante la ventaja relativa de exportación y la participación constante de mercado. La hipótesis del documento es que México es altamente competitivo en las exportaciones de tomate rojo y mantiene una alta concentración en el mercado estadounidense.

\section{Producción y comercio internacional de tomate rojo}

La producción de tomate a nivel mundial ha presentado un crecimiento importante de alrededor de $22 \%$ anual durante el periodo de 2003 a 2017. Los principales países productores son China (23\%), China Continental (22\%) y en menor participación la India (7\%), Estados Unidos de América (7\%) y Turquía (6\%) (Figura 1). Este grupo de países concentra el 64\% de la producción mundial de tomate. Por su parte, México contribuye con $2 \%$ de la producción mundial y ocupa la posición número 11 dentro de los países productores de tomate.

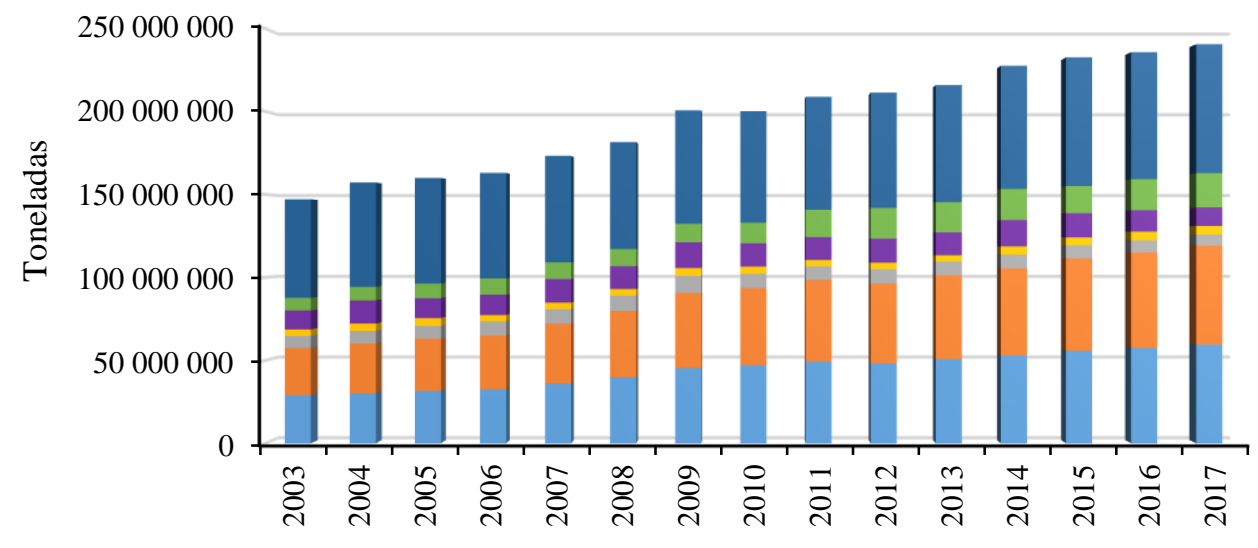

Año

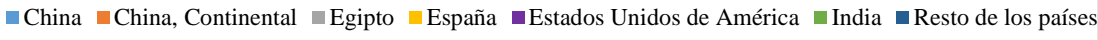

Figura 1. Principales productores de tomate rojo 2003-2017 (toneladas). Elaboración con datos de FAOSTAT.

Fideicomisos Instituidos en Relación con la Agricultura (FIRA), menciona que China e India destacan por su dinamismo en la producción mundial y esto se debe en parte a que China incrementó la productividad, mientras que en la India se destinó mayor parte de la superficie sembrada a esta hortaliza (FIRA, 2017).

Las exportaciones mundiales de tomate han crecido en promedio $4 \%$ anual. México se ubica como el principal exportador a nivel mundial de tomate, participando con cerca de $19 \%$ del volumen de exportación durante el periodo de 2003-2017. Posteriormente, España y Países Bajos exportan aproximadamente $13 \%$ cada uno (Cuadro 1). 
Cuadro 1. Principales países exportadores de tomate (toneladas).

\begin{tabular}{cccccccc}
\hline Año & España & Jordania & Marruecos & México & Países Bajos & Turquía & Mundo \\
\hline 2003 & 946511 & 186517 & 179804 & 903384 & 690949 & 227400 & 4615576 \\
2004 & 1023028 & 237859 & 107365 & 895126 & 771848 & 235364 & 4950300 \\
2005 & 923907 & 285169 & 166570 & 900767 & 770750 & 250182 & 5072827 \\
2006 & 987260 & 304529 & 192353 & 103503 & 776496 & 304372 & 5778283 \\
2007 & 880630 & 386968 & 297593 & 107646 & 834589 & 372094 & 6459293 \\
2008 & 938596 & 393983 & 346222 & 1042727 & 839550 & 439729 & 6547256 \\
2009 & 829540 & 431713 & 410118 & 1136299 & 923954 & 542259 & 6962655 \\
2010 & 738773 & 371257 & 372112 & 1509616 & 943119 & 574279 & 7190079 \\
2011 & 964054 & 434830 & 392435 & 1493316 & 1023496 & 576573 & 7562419 \\
2012 & 908755 & 418516 & 443811 & 1472390 & 977664 & 560430 & 7440702 \\
2013 & 1004009 & 611519 & 457854 & 1535157 & 1013529 & 483046 & 7864153 \\
2014 & 958272 & 517207 & 485421 & 1537946 & 1021484 & 585202 & 8485605 \\
2015 & 949366 & 419287 & 417332 & 1560588 & 1010843 & 541355 & 8137967 \\
2016 & 911106 & 361439 & 524907 & 1748858 & 992601 & 485963 & 8576288 \\
2017 & 809612 & 282271 & 527724 & 1742619 & 1089230 & 522876 & 8258502 \\
(\%) participación & $13 \%$ & $5 \%$ & $5 \%$ & $19 \%$ & $13 \%$ & $6 \%$ & $100 \%$ \\
TMCA & $-1 \%$ & $3 \%$ & $7 \%$ & $4 \%$ & $3 \%$ & $6 \%$ & $4 \%$ \\
\hline
\end{tabular}

Elaboración con información de FAOSTAT.

Sin embargo, al estimar la tasa media de crecimiento anual, se observa que Marruecos y Turquía crecieron por encima del promedio mundial de exportaciones de tomate (4\%). El primero presentó una tasa de crecimiento de $7 \%$ y Turquía $6 \%$. Por su parte, México creció al mismo ritmo de las exportaciones mundiales; mientras que España mostró un decrecimiento de $1 \%$ en las exportaciones de tomate (FAOSTAT, 2020).

Los principales importadores de tomate durante el periodo de análisis fueron Estados Unidos de América (21\%), Alemania (11\%), Federación Rusa (9\%), Francia (8\%) y Reino Unido de Gran Bretaña (6\%). Mientras que Federación Rusia y Estados Unidos de América crecieron en promedio anual 6\% y 4\%, respectivamente (FAOSTAT, 2020). A pesar de que Estados Unidos de América, sobresale como uno de los principales productores a nivel mundial, su demanda es mayor, por lo que requiere complementar el consumo aparente con importaciones

\section{Producción de tomate rojo en México}

En México la mayoría de las entidades federativas producen tomate rojo, sin embargo, ocho estados concentran $72.02 \%$ de la producción nacional (SIAP, 2020). El principal productor es Sinaloa, que contribuyó durante el periodo de 2003-2017 con más de 32\% a nivel nacional (Cuadro 2). Posteriormente Baja California (8.25\%), Michoacán (7.05\%), San Luis Potosí (6.62\%), Jalisco (5.43\%), Zacatecas (4.73\%), Baja California Sur (4.54\%) y con menor participación Sonora (3.12\%) (SIACON, 2020). 
Cuadro 2. México: volumen de la producción de tomate rojo, 2003-2017 (toneladas).

\begin{tabular}{|c|c|c|c|c|c|c|c|c|c|c|}
\hline 运 & ص & 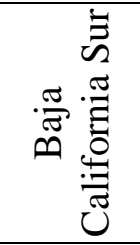 & 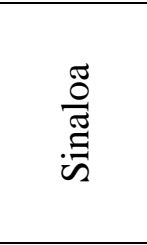 & 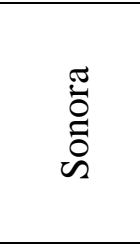 & 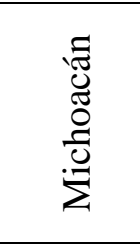 & 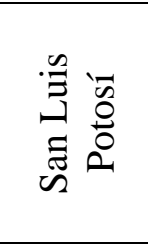 & 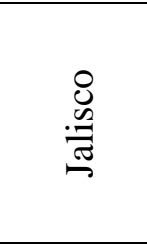 & 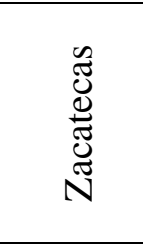 & 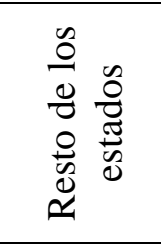 & $\begin{array}{l}\bar{\Xi} \\
\tilde{0} \\
\frac{0}{\tilde{z}} \\
z\end{array}$ \\
\hline 2003 & 7 & 122045 & 2685 & 3377 & 237454 & 208799 & 24 & 58939 & 379335 & 217 \\
\hline 2004 & 294076 & 113450 & 991113 & 51339 & 162476 & 125123 & 109930 & 37333 & 429790 & 2314630 \\
\hline 2005 & 262458 & 7954 & 845477 & 47412 & 150730 & 162053 & 117500 & 70347 & 472316 & 2246246 \\
\hline 2006 & 6000 & 114462 & 314 & 73096 & 134178 & 120120 & 87534 & 10 & 45 & 2093432 \\
\hline 2007 & 196388 & 132873 & 827011 & 63609 & 224898 & 120289 & 141796 & 112980 & 605559 & 2425403 \\
\hline 2008 & 206257 & 1 & 782910 & 43994 & 175703 & 139653 & 122421 & 76199 & 604038 & 2263202 \\
\hline 2009 & 180135 & 1 & 8303 & 41400 & 140185 & 112150 & 11 & 95 & 588370 & 3815 \\
\hline 2010 & 221626 & 1032 & 687057 & 60131 & 155354 & 98093 & 140802 & 14 & 667155 & 2277791 \\
\hline 2011 & 162325 & 92 & 011 & 60718 & 3081 & 108614 & 10 & 13 & 683941 & 2482 \\
\hline 2012 & 189636 & 10 & 9368 & 82324 & 171039 & 116137 & 60 & & 217 & 8370 \\
\hline 2013 & 196453 & 108 & 983,288 & 91856 & 98435 & 141108 & 134437 & 14 & 796454 & 2694358 \\
\hline 2014 & 135741 & 1 & 867832 & 387 & 169769 & 196011 & 1 & 92 & 33 & 5164 \\
\hline 2015 & 0848 & & 19342 & 136045 & 22 & 22 & & & & 29 \\
\hline 2016 & 226062 & 135223 & 924153 & 128038 & 235,7 & 306621 & 158 & & 387 & 3349154 \\
\hline 2017 & 179574 & 99379 & 937796 & 118526 & 253576 & 340836 & 219134 & 193363 & 112 & 3469707 \\
\hline TMCA & -2.21 & -1.36 & 1.57 & 3.74 & 0.44 & 3.32 & 5.2 & 8.24 & 7.53 & 3.17 \\
\hline $\begin{array}{c}(\%) \text { de } \\
\text { participació } \\
2003-2017\end{array}$ & 8.25 & 4.54 & 32.27 & 3.12 & 7.05 & 6.62 & 5.43 & 4.73 & 27.98 & 100 \\
\hline
\end{tabular}

Elaboración con datos obtenidos de SIAP.

A pesar de que el principal productor es Sinaloa, éste mantiene un crecimiento menos significativo en comparación con Zacatecas y Jalisco que presentaron un crecimiento promedio anual de $8 \%$ y $5 \%$. De acuerdo con Macías (2010), la pérdida de productividad en Sinaloa se debe principalmente por la escasez de agua y crecimiento de plagas. Mientras que Zacatecas ha intensificado la utilización de sistemas de producción bajo agricultura protegida de manera expansiva (Padilla et al., 2008). De igual forma, Sonora, Michoacán y San Luis Potosí crecieron en menor proporción que la media nacional.

En tanto que los estados de Baja California y Baja California Sur, presentaron tasas de crecimiento negativas de $2.21 \%$ y $1.36 \%$ durante el periodo de 2003 y 2017. Estas dos últimas entidades ubicadas en zonas áridas tienen limitaciones de recursos hídricos, debido a la sobreexplotación de sus acuíferos y escasa precipitación, lo que originan que la disponibilidad y calidad del agua sea insuficiente para la producción agrícola, elementos primordiales para el desarrollo de la actividad (Velasco et al., 2019). Algunos estados como Baja California, Baja California Sur, Jalisco y Michoacán se establecieron como operación de empresas sinaloenses que buscaban complementar la oferta, desestacionalizar la producción y mayor acceso de las exportaciones para aprovechar el mercado norteamericano en crecimiento (Avendaño, 2008; FIRA, 2019). 
Durante el periodo de 2003 a 2017, se encontró que estos mismos estados mostraron un crecimiento en el valor de la producción de aproximadamente $6 \%$ y mantuvieron un precio pagado al productor por arriba del promedio nacional $\left(\$ 6281.00 \mathrm{t}^{-1}\right)$, sobresaliendo Baja California con un precio por arriba de 50\% del nacional (Figura 2).

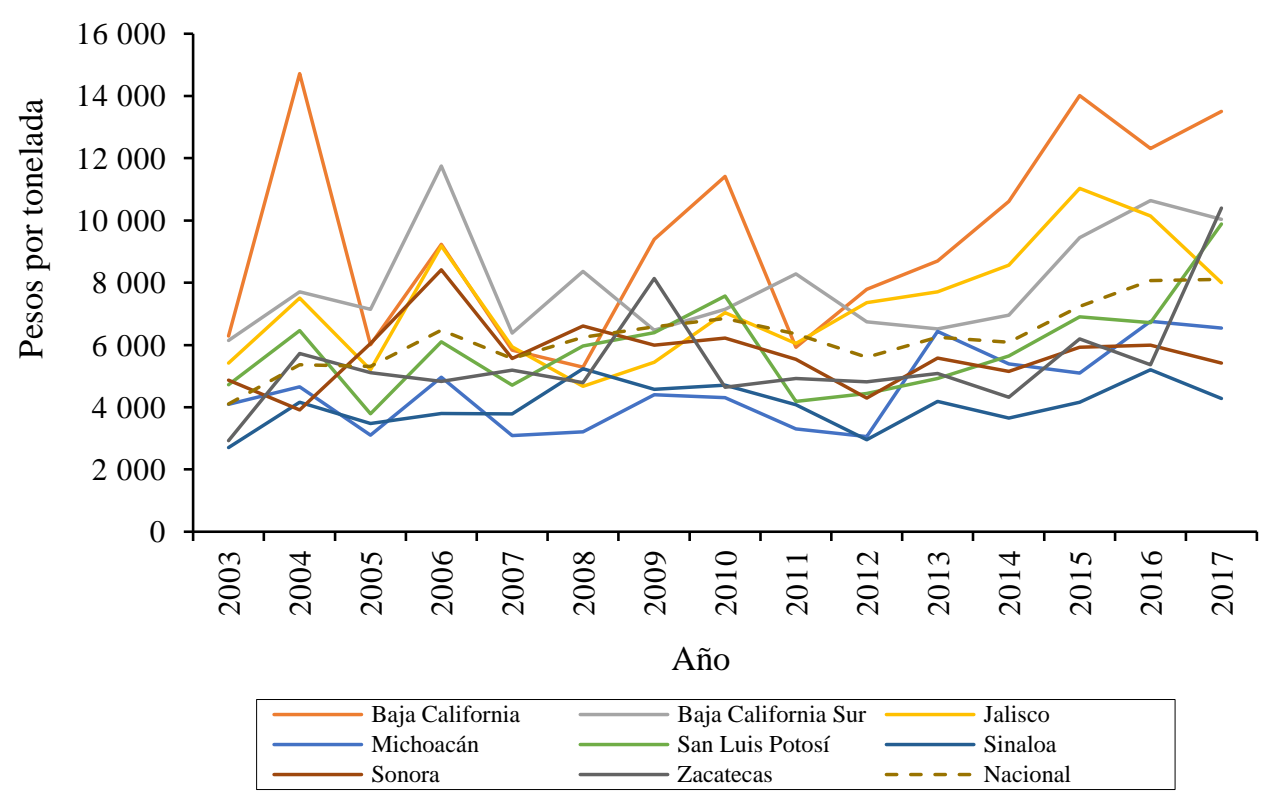

Figura 2. México: precio medio rural de tomate rojo, 2003-2017 (pesos por toneladas). Elaboración propia con datos de SIAP.

\section{Materiales y métodos}

Para analizar la competitividad del tomate rojo mexicano en el mercado de EE. UU., se utilizaron dos métodos: índice de ventaja relativa de exportación (VRE) propuesto por Vollrath (1989) y el método de análisis de participación constante en el mercado (CMS por sus siglas en inglés) conforme al planteamiento de Ahmadi-Esfahani (1995). Para ello, se obtuvo información estadística de la FAOSTAT y de ERS-USDA.

\section{Índice de ventaja relativa de exportación (VRE)}

El índice VRE propuesto por Vollrath es, en realidad, un replanteamiento del índice propuesto por Ballasa denominado ventaja comparativa revelada (RCA en inglés) (Laursen, 1998). El índice (VRE) refleja que determinado país tiene una ventaja relativa de exportación en un producto si (VRE) es positivo o mayor que 1, mientras indica una desventaja relativa de exportación si (VRE) es negativo o menor que 1. En términos generales, el (VRE) indica que a medida que su magnitud aumenta, un país se considera más especializado y con mayor competitividad. El índice VRE se define de la siguiente manera: VREai= (Xai/Xni)/(Xar/Xnr). En donde: VREai= ventaja relativa de exportaciones del producto a en el país i. Xai= valor de las exportaciones del producto a en el país $\mathrm{i}$. Xni= valor de las exportaciones totales en el país i sin incluir el producto a. Xar= valor de las exportaciones totales del producto a en el mundo, sin incluir al país i. Xnr= valor de las exportaciones totales en el mundo sin incluir el producto a y el país i. 


\section{Análisis de participación constante en el mercado (CMS)}

El método CMS, permite explicar el cambio en las exportaciones de un producto determinado a partir de sus componentes estructural y de competitividad. Este método fue propuesto por Leamer y Stern, posteriormente se adaptó para ampliar el análisis del crecimiento de las exportaciones (Avendaño, 2008). Ahmadi-Esfahani (1995), descompone el cambio en las exportaciones en dos niveles: el primer nivel de descomposición se realiza conforme a la siguiente igualdad: $\Delta \mathrm{q}=\mathrm{Sj} 0 \Delta \mathrm{Qj}$ $+\Delta \mathrm{SjQj} 0+\Delta \mathrm{Sj} \Delta \mathrm{Qj} . \mathrm{Sj} 0 \Delta \mathrm{Qj}=$ efecto estructural, refleja el cambio esperado en las exportaciones si se mantiene constante la participación inicial del país en el mercado mundial y en el país de destino.

Si este componente es positivo, significa que el incremento en la demanda por el producto favorece el incremento de las exportaciones. $\triangle \mathrm{SjQj} 0=$ efecto competitividad o residual, refleja la parte del cambio en las exportaciones que puede ser atribuido a un incremento en la competitividad en el periodo de análisis. Si este componente es positivo, significa que la competitividad ha aumentado, mientras que si el signo es negativo entonces se interpreta como una pérdida de competitividad. $\Delta \mathrm{Sj} \Delta \mathrm{Qj}=$ efecto interacción o de segundo orden, refleja el efecto de un cambio tanto en la participación de mercado como en la demanda en el cambio de las exportaciones.

El segundo nivel de descomposición se realiza mediante la obtención de seis efectos adicionales a partir de los componentes descritos en el primer nivel de descomposición. En este caso la igualdad es la siguiente: $\Delta \mathrm{q}=\mathrm{St} 0 \Delta \mathrm{Qj}+(\mathrm{Sj} 0 \Delta \mathrm{Qj}-\mathrm{St} 0 \Delta \mathrm{Qj})+\Delta \mathrm{StQj} 0+(\Delta \mathrm{SjQj} 0-\Delta \mathrm{StQj} 0)+(\mathrm{QtI} / \mathrm{Qt0}-\mathrm{I})$ $(\Delta \mathrm{SjQj} 0)+(\Delta \mathrm{Sj} \Delta \mathrm{Qj}-(\mathrm{QtI} / \mathrm{Qt} 0-\mathrm{I})(\Delta \mathrm{SjQj} 0)) . \mathrm{St} 0 \Delta \mathrm{Qj}=$ efecto crecimiento, refleja la parte del incremento en las exportaciones que se debe a un incremento en la demanda mundial mientras la participación del país exportador en el mercado mundial permanece constante.

$(\mathrm{Sj} 0 \Delta \mathrm{Qj}-\mathrm{St} 0 \Delta \mathrm{Qj})=$ efecto mercado, mide el cambio adicional en las exportaciones, mientras el exportador mantiene constante su participación en el mercado meta. Si el signo es positivo implica una concentración de las exportaciones en un mercado determinado. $\Delta \mathrm{StQj} 0=$ efecto residual puro, refleja el cambio en las exportaciones que ocurriría debido a un cambio en la competitividad general. $\triangle \mathrm{SjQj0}-\Delta \mathrm{StQj} 0=$ efecto residual estructural estático, éste refleja el cambio en las exportaciones que se atribuye a un cambio en la competitividad en un mercado determinado. QtI/Qt0-I $(\Delta \mathrm{SjQj} 0)=$ efecto de segundo orden puro ya que mide la interacción entre el cambio en la participación del exportador en el mercado meta y el cambio en la demanda mundial. $\Delta \mathrm{Sj} \Delta \mathrm{Qj}$ $(\mathrm{QtI} / \mathrm{Q} \mathrm{t} 0-\mathrm{I})(\Delta \mathrm{SjQj0})=$ efecto residual estructural dinámico, refleja la interacción entre el cambio en la participación del exportador en el mercado meta con el cambio en la demanda de dicho mercado.

\section{Resultados y discusión}

La competitividad de un producto en el mercado internacional depende de sus ventajas comparativas y está en función de los costos de producción, que incluyen estructura, costos de transporte y comercialización. Considerando que ésta se ve afectada por factores tales como, la calidad del producto, estacionalidad de la producción y mercado, grado de diferenciación y políticas gubernamentales tanto del país exportador como importador (Contreras-Castillo, 1999). Para Sánchez-Gómez et al. (2019) México basa sus ventajas comparativas en mano de obra y exceso de oferta de tierras con potencial productivo. 
Durante el periodo de análisis 2003-2017, las exportaciones mexicanas de tomate crecieron en promedio anual de $4 \%$, concentrando $98 \%$ al mercado estadounidense. Siendo este último el importador a nivel mundial, absorbiendo $21 \%$ de las importaciones mundiales de tomate (FAOSTAD, 2020). Sánchez-Gómez et al. (2019) mencionan que en 2013, las exportaciones de tomate mexicano hacia el mercado estadounidense ocupaban el $32.61 \%$ de las exportaciones totales.

El índice de VRE de tomate rojo de México en el mercado estadounidense presentó un valor positivo, es decir, muestra competitividad a lo largo del periodo 2003-2017, además las exportaciones de tomate rojo mostraron una tasa promedio de crecimiento anual de 6\% (Figura 3). Su competitividad se debe principalmente a la ventaja comparativa influyendo los factores de cercanía geográfica, precio, calidad y la temporalidad de la producción de tomate en Estados Unidos de América que desciende en el invierno (Hernández et al., 2004). Además, se deriva del proceso de integración entre México y Estados Unidos de América (Macías, 2003).

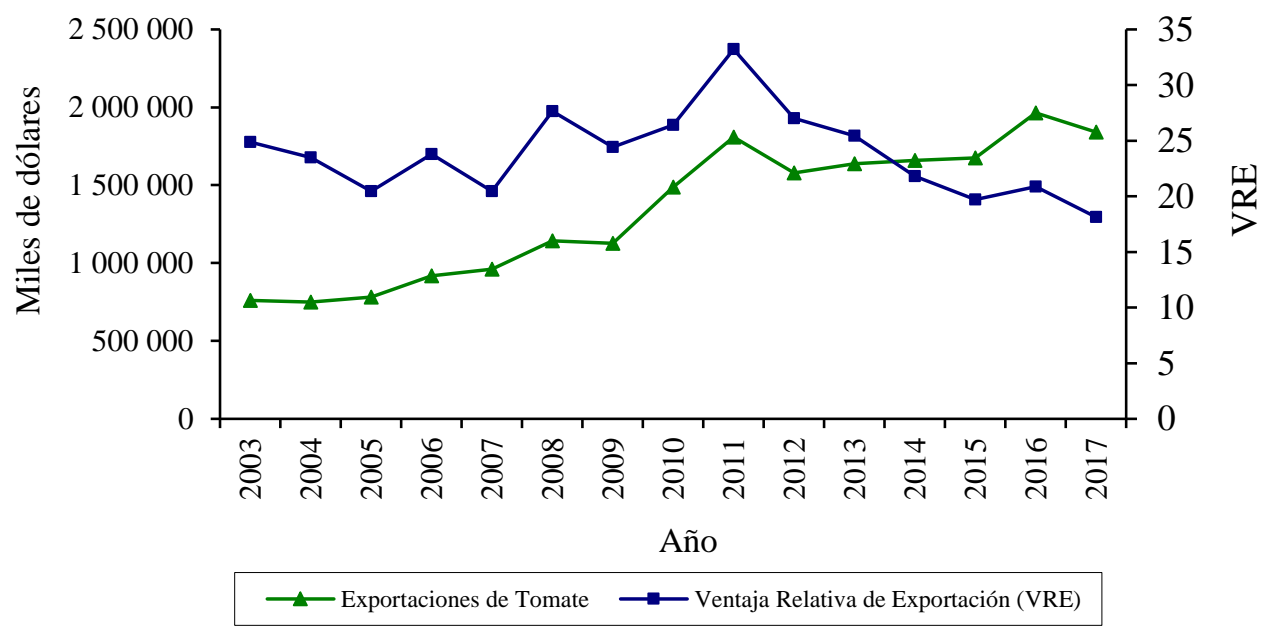

Figura 3. México: exportaciones de tomate e índice de ventaja relativa de exportación en el mercado estadounidense, 2003-2017. Elaboración con información de FAOSTAT.

A pesar de que el indicador VRE es mayor a 1, y mientras más alto sea, se considera más especializado, en los últimos años, a partir de 2011 la competitividad del tomate rojo mexicano en el mercado de estadounidense ha decrecido, pasando de 33 a 18 el índice de VRE. Pese a que la competitividad es positiva, muestra una tendencia a la baja. Lo que estaría indicando que México cede su participación en el mercado a otros países y que pierde especialización en este subsector. De acuerdo con Avendaño et al. (2006), Canadá se está especializando en la producción de tomate de invernadero. Lo anterior, puede obedecer a las diferentes políticas comerciales y fitosanitarias impuestas por Estados Unidos de América, por varios motivos el primero, derivado de que en algunos años se dieron brotes epidemiológicos asociados a frutas y hortalizas, entre ellos el tomate.

Como respuesta a estos brotes, en 1997, se diseñó Produce and Imported Food Safety Iniciative (iniciativa de inocuidad alimentaria en productos domésticos e importados) (Avendaño et al., 2006). En segundo, originado por la imposición de aranceles a las exportaciones de tomate mexicano con la finalidad de proteger a los productores de Florida, principal zona productora de tomate en ese país o por las acusaciones de prácticas desleal de comercio, dumping en contra de los productores mexicanos de tomate (Avendaño y Acosta, 2009; CEDRSSA, 2018). 
Asimismo, Soto (2018) encontró que México muestra competitividad en las exportaciones de tomate durante el periodo de 1994 a 2016 al analizar el índice de ventaja comparativa revelada. Además, señala que México mantiene niveles de competitividad superiores a sus socios comerciales del TLCAN y China. Por su parte, Crespo y Plzákova (2018) en su estudio concluyen que las exportaciones de tomate mexicano en el mercado estadounidense es el más competitivo y con tendencia positiva en comparación con Guatemala, Canadá, República Dominicana y Holanda.

\section{Participación constante de mercado (CMS)}

El CMS muestra la competitividad y comportamiento de las exportaciones en el mercado destino. Se estimaron el primer y segundo orden de descomposición para los principales países exportadores de tomate rojo en el mercado de Estados Unidos de América, donde México concentra alrededor de $89 \%$. Canadá exporta $10 \%$ y con menor participación, pero durante el periodo han mantenido sus exportaciones República Dominicana, Guatemala y Países Bajos (Figura 4).

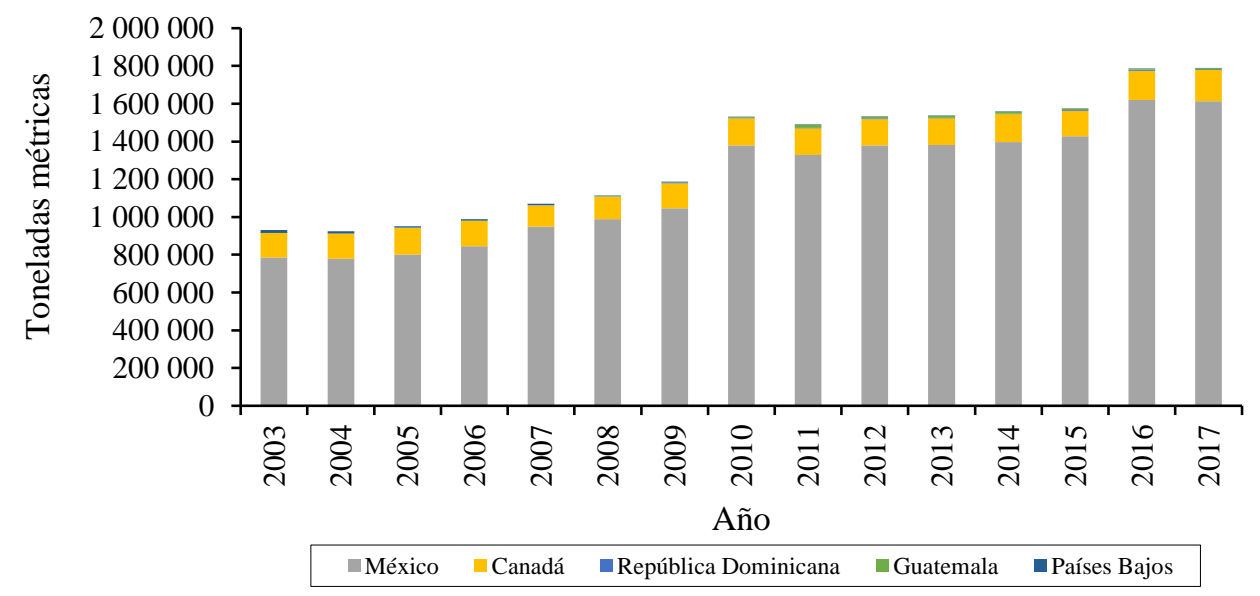

Figura 4. Principales países exportadores de tomate a Estados Unidos de América, 2003-2017 (toneladas métricas). Elaboración con datos de ERS-USDA.

Los resultados de la aplicación del método CMS se presentan en el Cuadro 3. El primer aspecto que destaca es que el cambio en el volumen de exportaciones fue positivo para México, Canadá, República Dominicana y Guatemala, mientras que para Países Bajos es negativo.

Cuadro 3. Participación constante de mercado de las exportaciones de tomate rojo en el mercado de Estados Unidos de América, 2003-2017.

\begin{tabular}{cccccc}
\hline Concepto & México & Canadá & $\begin{array}{c}\text { República } \\
\text { Dominicana }\end{array}$ & Guatemala & Países Bajos \\
\hline \multicolumn{5}{c}{ Efecto primer orden } \\
Cambio en exportaciones & 757106.78 & 21857.21 & 5648.55 & 5139.44 & -13564.91 \\
Efecto estructural & 654934.65 & 108565.28 & 17.52 & 1.08 & 12668.53 \\
Efecto competitividad & 53229.55 & -45173.11 & 2933.65 & 2676.97 & -13667.08 \\
Efecto segundo orden & 48942.57 & -41534.97 & 2697.38 & 2461.38 & -12566.36 \\
\hline
\end{tabular}


Rev. Mex. Cienc. Agríc. vol. 12 núm. 728 de septiembre - 11 de noviembre, 2021

\begin{tabular}{cccccc}
\hline Concepto & México & Canadá & $\begin{array}{c}\text { República } \\
\text { Dominicana }\end{array}$ & Guatemala & Países Bajos \\
\hline \multicolumn{5}{c}{ Efecto segundo orden } \\
Efecto crecimiento & 400927.52 & 58338.34 & 202.82 & 10070.85 & 306647.53 \\
$\quad$ Efecto mercado & 254007.13 & 50226.94 & -185.3 & -10069.77 & -293979 \\
$\quad$ Efecto residual puro & 57158.4 & -9127.17 & 2174.57 & 3373.38 & -53579.17 \\
$\begin{array}{c}\text { Efecto residual estructural } \\
\text { estático }\end{array}$ & -3928.84 & -36045.93 & 759.09 & -696.4 & 39912.09 \\
$\begin{array}{c}\text { Efecto segundo orden puro } \\
\text { Efecto residual estructural } \\
\text { dinámico }\end{array}$ & 37875.05 & -32142.55 & 2087.42 & 1904.78 & -9724.69 \\
\hline
\end{tabular}

Elaboración con datos de FAOSTAD y ERS-USDA.

En el primer nivel de descomposición, el efecto estructural es positivo para todos los países, lo que refleja crecimiento de la demanda de tomate que favorece las exportaciones, principalmente para México. Crespo y Plzákova (2018) realizaron un estudio seleccionando los mismos países, obteniendo que el efecto estructural es positivo para todos los países, donde México tiene mayor ventaja dada su proximidad geográfica con el mercado estadounidense. En otro estudio Avendaño y Acosta (2009), encontraron que el efecto estructural es positivo para los países exportadores de tomate que se seleccionaron para el mercado estadounidense (Canadá, España, Israel, México y Países Bajos) y señalan un impacto positivo en la demanda mundial de tomate.

En el caso del efecto competitividad, el signo es positivo, principalmente para México y en menor impacto para República Dominicana y Guatemala, mostrando una pérdida para Canadá y Países Bajos. El efecto segundo orden muestra la influencia de la participación de mercado y los cambios en la demanda de las exportaciones de tomate rojo, es positivo para México, República Dominicana y Guatemala derivado del comportamiento en la competitividad. El incremento en la demanda mundial de tomate rojo creció en promedio $4 \%$ anualmente del volumen de importaciones (FAOSTAT, 2020), provocando un impacto positivo en el efecto crecimiento para todos los países durante este periodo de análisis.

Avendaño y Acosta (2009) indican resultados similares, al encontrar que el efecto crecimiento es positivo para todos los países, favoreciendo principalmente a Países Bajos; sin embargo, las exportaciones de éste y de México han sido desplazadas por Canadá. El efecto mercado es positivo para México y Canadá, lo que muestra que ambos concentran sus exportaciones hacia un solo mercado. En el caso específico de México exporta alrededor de 98\% del volumen de tomate rojo hacia ese destino. Mientras que, para Guatemala, República Dominicana y Países Bajos el signo es negativo relacionado con diversificación de mercados; por ejemplo, Guatemala, en 2017, exportó a El Salvador 44073 toneladas y solo 6221 toneladas a Estados Unidos de América. En México se concentra más de $90 \%$ de las exportaciones al mercado estadounidense generando vulnerabilidad a los productores mexicanos al someterse a las condiciones del mercado de los Estados Unidos de América, cambios en los patrones de compra y consumo y situación económicas del país.

El efecto residual puro es positivo para México, Guatemala y República Dominicana mostrando un aumento de competitividad. A pesar de ello, el efecto residual estructural estático arroja un signo positivo para República Dominicana y Países Bajos, indicando una mejora en su competitividad, 
generando mayor participación en el mercado mundial. Por último, los efectos de segundo orden puro y el residual estructural dinámico presentan signo positivo para México, República Dominicana y Guatemala, lo que significa que los países exportadores de tomate han sido capaces de aumentar su participación en el mercado estadounidense, al mismo ritmo en que estuvo creciendo la demanda mundial y la demanda en el mercado destino.

\section{Conclusiones}

México es un país altamente competitivo en la exportación de tomate rojo en el mercado de Estados Unidos de América, derivado de su ubicación geográfica, el uso de innovaciones tecnológicas, disponibilidad de tierra, mano de obra, entre otros factores que se relacionan con la ventaja comparativa. La poca diversificación de mercados de las exportaciones mexicanas de tomate muestra una vulnerabilidad al estar sujetas a las barreras arancelarias y no arancelarias, impuestas por el mercado destino para apoyar y proteger a los productores locales.

A pesar de que México, durante el periodo de análisis ha mostrado competitividad, es importante diseñar estrategias de comercialización que le permitan explorar nuevos destinos y mantener la participación de las exportaciones. Para ello, se sugiere que los productores mexicanos aprovechen los acuerdos comerciales que se tiene firmados con diferentes países, diferenciación del producto a través sus variedades y formas de producción, además de introducirse en el mercado de productos procesados del tomate.

\section{Literatura citada}

Ahmadi, F. Z. 1995. Wheat market share in the presence of Japanese import quotas. J. Policy Model. 3(17):315-323.

Álvarez, M. M. T.; Núñez, R. M. A. y Wendladt, A. T. R. 2017. Caracterización de la cadena de valor del tomate rojo fresco en México. Rev. Glob. Negoc. 3(5):45-58.

Avendaño, R. B. D. 2008. Globalización y competitividad en el sector hortofrutícola: México el gran perdedor. México. Rev. Cotid. 147:91-98.

Avendaño, R. B. D. y Acosta, M. A. I. 2009. Midiendo los resultados del comercio agropecuario mexicano en el contexto del TLCAN. Rev. Estud. Socia. 33(17):41-81.

Avendaño, R. B. D.; Rindemann, S. R.; Lugo, M. S. Y. y Mungaray, L. A. 2006. La inocuidad alimentaria en México. Las hortalizas frescas de exportación. UABC-Miguel Ángel Porrúa. $1^{\mathrm{a}}$. (Ed.). Mexicali, Baja California, México. 226 p.

Avendaño, R. B. y Schwentesius, R. R. 2012. El sector hortofrutícola mexicano de exportación: acciones y políticas para su fortalecimiento. In: políticas agropecuarias, forestales y pesqueras. Análisis estratégico para el desarrollo. Calva, J. L. (Coord.). 9. Juan Pablos (Ed.). México, DF. 305-319.

Avendaño, R. B. y Schwentesius, R. R. 2005. Factores de competitividad en la producción y exportación de hortalizas: el caso del Valle de Mexicali, BC. México. Rev. Latinoam. Econ. Prob. Des. 140(36):165-192.

Ávila, A. D. y González, M. D. 2012.La competitividad de las fresas (Fragaria spp.) mexicanas en el mercado nacional, regional y de Estados Unidos. México. Rev. Agric. Soc. Des. 1(9):17-27. 
Ávila, A. D.; González, M. D.; Montaño, M. I. E. y Vizcaíno, V. L. A. 2020. Análisis de la competitividad de nuez pecanera mexicana en el mercado internacional. Rev. Mex. Cienc. Agríc. 4(11):895-904.

Bracamontes, A. y Méndez, R. 2011. Subvenciones y reconversión productiva. El caso del trigo en Sonora. México. El colegio de sonora. Hermosillo, Sonora, México. 204 p.

CEDRSSA. 2018. Centro de Estudios para el Desarrollo Rural Sustentable y la Soberanía Alimentaria. La producción y el comercio del tomate en México. http://www.cedrssa.gob.mx /files/b/13/86Tomate.pdf.

CEDRSSA. 2019. Centro de Estudios para el Desarrollo Rural Sustentable y la Soberanía Alimentaria. Reporte resultados de la encuesta nacional agropecuaria 2017. http://www.cedrssa.gob.mx/files/b/13/37ResultadosENA2017.pdf.

Contreras, C. J. M. 1999. La competitividad de las exportaciones mexicanas de aguacate: un análisis cuantitativo. México. Rev. Chapingo. Ser. Hortic. 5:393-400.

Crespo S. L. and Plzákova L. 2018. Production of tomatoes in Mexico and its competitiveness in the U.S. market. 171-179. 10.36689/uhk/hed/2018-01-016.

FIRA. 2017. Fideicomisos Instituidos en Relación con la Agricultura. Panorama Agroalimentario, tomate rojo 2017. https://www.inforural.com.mx/fira-panorama-agroalimentario-tomaterojo-2019/.

FAOSTAT. 2020. Organización de las Naciones Unidas para la Alimentación y la Agricultura. Estadísticas. http://www.fao.org/faostat/es/\#home.

Hernández, M. J.; García, M. R.; Valdivia, A. R. y Omaña, S. J. M. 2004. Evolución de la competitividad y rentabilidad del cultivo del tomate rojo (Lycopersicon esculentum) en Sinaloa, México. Agrociencia. 38:431- 436.

Hernández, P. L. 2019. Desarrollo tecnológico e integración comercial de los productores agrícolas de la Costa de Hermosillo en la globalización. México. Rev. Reg. Socie. 31:1-25.

INEGI. 2007. Instituto Nacional de Estadística y Geografía. Censo Agrícola, Ganadero y Forestal 2007. https://www.inegi.org.mx/programas/cagf/2007/.

Krugman, R. P. y Obstfeld, M. 2006. Economía internacional. $7^{a}$ (Ed.). Pearson Education.

Laursen, K. 1998. Revealed comparative advantage and the alternative as measures of international specialization. Danish research unit for industrial dynamics, working paper. 98-30 pp.

Macias, M. A. 2003. Enclaves agrícolas modernos: el caso del jitomate mexicano en los mercados internacionales. México. Región y Sociedad. 26(15):103-151.

Macías, M. A. 2010. Competitividad de México en el mercado de frutas y hortalizas de Estados Unidos de América, 1989-2009. Rev. Agroalimentaria. 31(16):31-48.

Padilla, B. L. E.; Rumayor, R. A. F. y Pérez, V. O. 2008. La competitividad sistémica de la industria del tomate de agricultura protegida en Zacatecas. México. Rev. Merc. Negoc. 9(17):38-59.

Pugel, T. A. 2004. Economía internacional. 12a (Ed.). Mc Graw-Hill.

Ramírez, P. L. C.; Caamal, C. I.; Pat, F. V. G.; Martínez, L. D. y Pérez, F. A. 2020. Análisis de los indicadores de competitividad de las exportaciones de fresa mexicana. Rev. Mex. Cienc. Agríc. 4(11):815-827.

Ramírez, P. L. C.; Caamal, C. I.; Pat, F. V. G. y Martínez, L. D. 2016. Índices de competitividad de la fresa (Fragaria vesca L.) de México en el mercado mundial. Agroprod. 5(9):29-34.

SADER. 2021. Secretaría de Agricultura y Desarrollo Rural. Análisis de la balanza comercial agroalimentaria de México. https://www.gob.mx/cms/uploads/attachment/file/627936/ Balanza_ Comercial_Agropecuaria_ y_Agroindustrial_enero_2021.pdf. 
Sánchez-Gómez, C.; Caamal-Cauich, I. y del Valle-Sánchez, M. 2019. Exportación hortofrutícola de México hacia los Estados Unidos de América. Estudios Sociales. Rev. Aliment. Contemporánea y Desarrollo Regional. 29(54):1-20.

SIACON. 2020. Servicio de Información Agroalimentaria y Pesquera. Producción agrícola. https://www.gob.mx/siap/documentos/siacon-ng-161430.

SIAP. 2020. Sistema de Información Agroalimentaria y Pesquera. Producción agropecuaria y pesquera. https://www.gob.mx/siap/acciones-y-programas/produccion-agricola-33119.

Soto, H. D. 2018. Análisis de la competitividad del tomate mexicano en el contexto del TLCAN: escenario ante el mercado chino. Rev. Econ. Admin. 15(1):15-31.

Torres, P. V. H. 2009. La competitividad del aguacate mexicano en el mercado estadounidense. Revi. Geog. Agríc. 43:61-79.

Valencia, S. K. y Zetina, E. A. M. 2017. La cebolla mexicana: un análisis de competitividad en el mercado estadounidense, 2002-2013. Región y Sociedad. 29(70):133-153. https://doi.org/10.22198/rys.2017.70.a348.

Valencia, S. K. y Ávila, D. 2019. La competitividad del comercio de uva en el mercado internacional y en Estados Unidos: el caso de México y Perú. Rev. Cimexus. 14. 10.33110/cimexus140103.

Valencia, S. K. 2016. Competitividad de las exportaciones de café de Colombia, Guatemala y México hacia el mercado estadounidense (2001-2014). Rev. Ciencia ergo-sum. 23:239-246.

Velasco, A. L.; De la O. B. V.; Ruiz, C. J. S. y Méndez, H. S. 2019. Gestión del agua en empresas productoras de hortalizas del Valle de San Quintín, Baja California. Rev. Glob. Negoc. 7(3):87-94.

Vollrath, T. 1989. Competitiveness and protection in world agriculture. United States. Agriculture Information Bulletin. 567:1-9.

Vollrath, T. 1991. A theoretical evaluation of alternative trade intensity measures of revealed comparative advantage. Review of World Economics. 127(2):265-279. 